\title{
Distractor dinámico
}

\section{para el tratamiento de fracturas conminutas} de las articulaciones

\section{interfalángicas proximales. Análisis biomecánico}

\author{
L. Gil Santos ${ }^{(1,2,3)}$, J. Más Estellés ${ }^{(1)}$, C. Barrios Pitarque ${ }^{(3)}$ \\ (1) Hospital InTERmutual de LeVANTE \\ (2) Centro de Biomateriales, Universidad Politécnica de Valencia \\ (3) Universidad Católica de Valencia. San Vicente Mártir.
}

Correspondencia:

Dr. Luis Gil Santos

Hospital Intermutual de Levante

Autovía Valencia Ademúz, km 11,7

46184 San Antonio de Benagéber (Valencia)

Teléfono 961350250

e-mail: lu.gils@telefonica.net

Se analiza el comportamiento mecánico de un distractor dinámico para el tratamiento de las fracturas conminutas de las articulaciones interfalángicas proximales (IFP) de los dedos. El objetivo es establecer cuáles son los patrones de la tendencia a luxarse, y por lo tanto saber dónde y cómo colocar las agujas de Kirschner correctamente. Metodológicamente se aplica un sistema de modelización matemática del comportamiento mecánico del dedo índice de la mano, con vistas a evaluar la mejor forma de movilizar, total o parcialmente, la articulación IFP, y conseguir la restauración de la superficie articular. Los resultados tienen importantes aplicaciones clínicas: en las lesiones con inestabilidad volar, cuanto más cerca de la articulación IFP se coloque la aguja, menor será la tendencia a la inestabilidad; en las lesiones con inestabilidad dorsal, se deberá colocar la aguja lo más distalmente posible.

Palabras clave: fijación externa, fractura-luxación, fractura de pilón, articulación IFP.
The mechanical behaviour of a dynamic distractor for the treatment of comminuted fractures of the proximal interphalangeal joints (PIP) of fingers is analyzed. The objective was to establish the patterns of dislocation tendency, and therefore to know where and how to place the Kirschner wires correctly. Methodologically, a system of mathematical modeling of mechanical behaviour of the index finger of the hand was used to assess the best option to mobilize (in whole or in part) the PIP joint, getting restoration of the articular surface. The results have important clinical applications: in unstable volar lesions, the closer to the PIP joint the needle is placed, the lower the tendency to instability; in lesions with dorsal instability, the needle should be placed as distally as possible.

Key words: external fixation, fracture-dislocation, pilon fracture, PIP joint. 


\section{INTRODUCCIÓN}

1 1 traumatismo articular más habitual de los dedos afecta a la articulación interfalángica proximal y en un alto porcentaje de los casos implica conminución de la base de falange media ${ }^{1}$. Un método de tratamiento habitual de estas fracturas consiste en la colocación de un distractor que permita el movimiento de la interfalángica proximal. Dicho distractor puede admitir varias posibilidades de inserción de las agujas de Kirschner (AK) condicionando la estabilidad articular. Dependiendo de la configuración del distractor se varían las posibilidades de giro del montaje y los efectos sobre la movilidad y estabilidad de la articulación IFP.

El objetivo de este trabajo es analizar un sistema de distracción dinámica y evaluar su efecto sobre la articulación IFP mediante un modelo bidimensional del movimiento de las tres falanges de uno de los dedos de la mano; el dedo elegido ha sido el índice. La filosofía del sistema que pretendemos analizar, consiste en la generación de fuerzas aprovechando la propia elasticidad de las AK para, transfiriéndola al hueso, conseguir una reducción y estabilización suficiente de la fractura que permita la movilidad precoz ${ }^{2-5}$. A tal fin se colocan dos agujas, una a la que llamaremos AK distractora (sometida a una deformación elástica cuya fuerza tendente a la recuperación aprovechamos) y una segunda, a la que llamaremos AK resistente, que nos posibilita la elección del punto de aplicación de las fuerzas y el giro de las agujas (Figura 1). Este sistema difiere del propuesto por Suzuki et al. ${ }^{6}$ en que la artrodiástasis se efectúa por la acción de unas bandas elásticas. En el sistema objeto de nues-

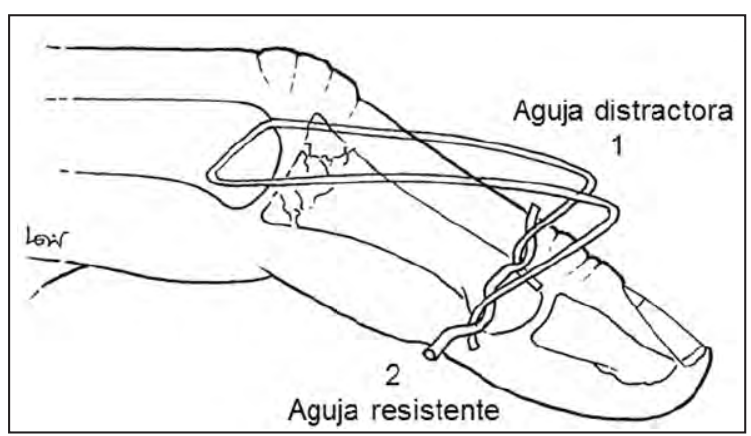

Figura 1. Configuración del sistema de distracción, 1. Aguja distractora. 2. Aguja resistente tro estudio la generación de la artrodiástasis se consigue gracia a la capacidad de recuperación elástica propia del material de las agujas. En el modelo matemático empleado en este trabajo, nos hemos basado en los datos antropométricos obtenidos por Chao et al. ${ }^{7}$.

Nuestra hipótesis es que variando el emplazamiento de las agujas variamos las fuerzas actuantes y por lo tanto la dirección de los desplazamientos. Si eso es así, ¿cuál es el emplazamiento idóneo para favorecer la estabilidad articular durante el movimiento? Con la modelización matemática que se expone a continuación nos preguntamos además si el modelo es capaz de predecir la tendencia a la luxación en distintas posiciones del dedo con distintos supuestos de esfuerzos, y cuál sería la localización idónea de las agujas.

\section{MATERIAL Y MÉTODO. GEOMETRÍA DEL MODELO}

El modelo bidimensional que se propone supone el dedo como un mecanismo formado por tres barras (las tres falanges) anclado a un elemento fijo (el metacarpo). Se admite que su movimiento sólo puede producirse en el plano formado por las tres falanges y la posición se define por los tres ángulos de giro de las tres articulaciones, las dos interfalángicas y la metacarpo-falángica.

Con el fin de caracterizar la posición del dedo en cualquier situación, se establecen siete sistemas de referencia (0-6): cada falange lleva asociados dos sistemas de referencia que se mueven con ella (Figura 2). De este modo, la posición de cada punto de una falange con respecto a cada uno de sus dos sistemas de referencia será siempre la misma, independientemente de la configuración geométrica del dedo. El último sistema de referencia (6), anclado al metacarpo, se considera como sistema fijo.

$\mathrm{El}$ origen del primer sistema de referencia de la falange distal (0) está situado en el extremo del dedo, y el origen del segundo (1) en el centro de la superficie cóncava articular de la articulación IFD. Ambos sistemas tienen sus ejes $\mathrm{X}$ alineados con el eje de la falange distal, con el sentido positivo apuntando hacia el extremo (distal), y 


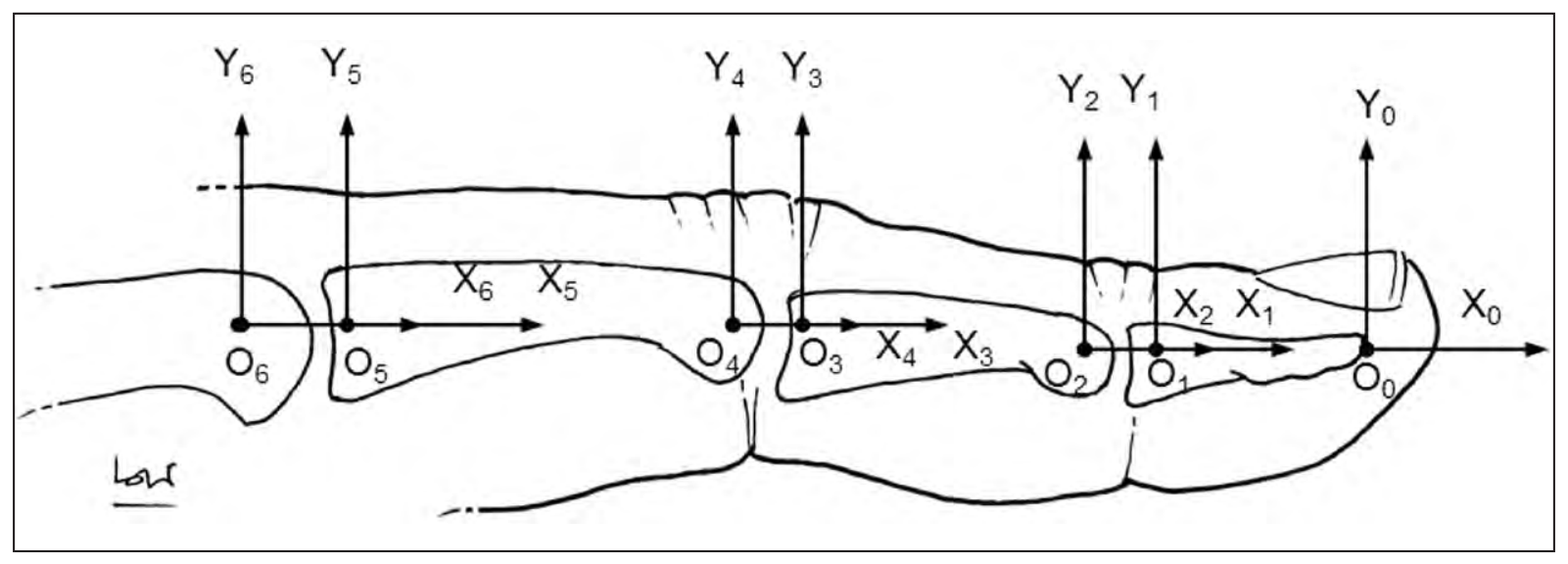

Figura 2. Sistemas de referencia de los dedos utilizados para la modelización de las ecuaciones de equilibrio de fuerzas y momentos, que permiten comprender las respuestas funcionales y mecánicas.

sus ejes Y son paralelos en el sentido dorsal de la mano. Como dichos sistemas se mueven solidariamente con la falange distal, siempre permanecerán paralelos (Figura 2). Los ejes Z son todos paralelos, perpendiculares al plano del movimiento del dedo y dirigidos hacia el lector.

En las otras falanges, el primer sistema de referencia (par) tiene su origen en el centro de giro de los cóndilos de la articulación correspondiente, y el segundo sistema de referencia (impar), en el centro de la superficie cóncava articular de la base de la falange distal correspondiente que configura la articulación.

Los parámetros que caracterizan la posición del dedo son: el ángulo de la falange proximal respecto del metacarpiano, el de la falange media respecto de la falange proximal, y el de la falange distal respecto de la falange media. Dichos ángulos, que en la figura 2 son todos nulos, se miden desde el eje $\mathrm{X}$ de cada sistema par hasta el eje $\mathrm{X}$ del sistema anterior (positivo en el sentido de las agujas del reloj).

El primer paso del modelo para evaluar la respuesta mecánica es dar valores a estos tres parámetros para establecer una posición del dedo. En principio, no hay restricciones sobre los valores que pueden adoptar dichos parámetros, pero obviamente, para obtener resultados verosímiles, deberían respetarse las limitaciones fisiológicas, aproximadamente entre 0 y $90^{\circ}$.

Las dimensiones de cada falange y las posiciones de centros de giro y de superficies cóncavas articulares han sido tomadas de Chao et al. ${ }^{7}$ a partir de un estudio estadístico antropométrico. Todos estos valores están normalizados al valor de la longitud de la falange media (distancia $\mathrm{O}_{2} \mathrm{O}_{3}$ ), que es tomada como unidad. Por este motivo, en todas las gráficas se representa la posición del distractor mediante magnitudes relativas y por tanto, adimensionales.

\section{Motores}

El movimiento del dedo es producido por las fuerzas generadas en los músculos correspondientes, que llegan a los dedos a través de los tendones respectivos, cuya función básica es transmitirlas y aplicarlas al hueso, en puntos concretos, permitiendo así el movimiento articular. Este modelo tan sólo considera los esfuerzos producidos por la musculatura extrínseca a la mano, no considerando la musculatura intrínseca. Los esfuerzos a la falange distal son transmitidos a través del tendón extensor digitorum en la parte dorsal del dedo), y del tendón flexor digitorum profundus en la parte palmar. Ambos tendones se insertan en puntos fijos de la falange, estando determinada la dirección de actuación de la fuerza en cada uno de dichos tendones. Tanto los puntos de inserción como las direcciones de aplicación de las fuerzas transmitidas se han tomado de Chao et al. ${ }^{7}$, siendo estos valores conocidos y determinados.

El movimiento de la falange media es transmitido por cinco tendones:

- Un tendón extensor, anclado a la falange media. 
- Dos bandeletas o cintillas laterales extrínsecas, que no se anclan a la falange media, sino que deslizan sobre el dorso de la cabeza de F1, que va a actuar como una polea durante la flexoextensión, y también a través de la cabeza de F2, que también actúa como una polea, acabando insertadas en la falange distal; ambas bandeletas se unen distalmente sobre el tercio distal de la falange media para dar lugar al tendón extensor de la falange distal; por ello, la suma de las fuerzas transmitidas por ambas bandeletas será la fuerza actuante en el tendón extensor de la falange distal. Además, como dichas bandeletas deslizan a través de las «poleas» del dorso de la cabeza de F1 y el dorso de F2, no ejercen ninguna fuerza en la dirección axial de la falange media, teniendo la fuerza, únicamente, componente en la dirección del eje Y. En aras a simplificar el modelo, y dado que la polea del tendón extensor se encuentra en el extremo distal de la falange media, donde dicho tendón extensor aún no se ha dividido para formar las dos bandeletas laterales, consideramos que toda la fuerza del tendón extensor es transmitida a través de la bandeleta radial, en el mencionado extremo distal de la falange media. El esfuerzo debido al tendón extensor en esta falange media es calculado teniendo en cuenta que en dicho punto se produce un cambio de dirección del esfuerzo. La consideración de esfuerzos en la bandeleta ulnar sólo afectaría al movimiento en un plano perpendicular al de estudio, que aquí no consideramos.

- Dos tendones flexores, uno flexor superficial insertado en la falange media y otro profundo, insertado en la falange distal. El flexor digitorum profundus desliza a través de una polea insertada en la falange media, por lo que no produce esfuerzo axial sobre dicha falange; el único esfuerzo producido por este tendón sólo tiene componente $\mathrm{Y}$, y deriva del cambio de dirección de la fuerza en la polea. El tendón flexor digitorum superficialis va anclado directamente a la falange media.

En cuanto a la falange proximal, existen 6 tendones que producen su movimiento, pero como el objetivo es analizar el distractor dinámico colocado entre la falange proximal y la falange media, no es necesario analizar los esfuerzos de estos seis tendones de la falange proximal. En cada articulación, cápsulas articulares y placas volares realizan los esfuerzos necesarios para mantener la integridad del mecanismo.

Dado que, en realidad, desconocemos la aportación de cada parte del aparato flexor y extensor durante la dinámica de la flexo-extensión digital «in vivo», el análisis supone ir anulando los esfuerzos aplicados por determinados tendones y calculando los esfuerzos originados en las articulaciones, esfuerzos que nos permiten conocer cuál sería la tendencia a la luxación. De esta forma, podemos observar y comprender cuál sería el comportamiento del sistema. Dicho de otro modo, cuáles serían las condiciones necesarias para que el sistema funcionara correctamente.

\section{Fundamento biomecánico de la distracción dinámica}

El sistema se fundamenta en la reducción y estabilización de fracturas articulares complejas mediante el principio de la ligamentotaxis. Aprovechando la elasticidad de las agujas de Kirschner, la fuerza es transmitida al hueso, y este a su vez lo hará a los fragmentos articulares a través de los ligamentos a ellos solidarizados. Gracias a ello y a la movilización, se consigue la restauración de una superficie articular aceptable. En resumen, con el análisis de los resultados de nuestro modelo, pretendemos identificar las posibles acciones de mejora y optimizar el distractor dinámico.

\section{Proceso de cálculo}

El proceso de cálculo comienza asignando un valor a cada uno de los ángulos de las tres articulaciones consideradas dando una configuración geométrica al dedo. Una vez establecida la posición a estudiar, se considera cada una de las tres falanges separadamente, identificando todas las fuerzas y momentos actuantes en cada una de ellas. Estas fuerzas y momentos pueden provenir tanto de las transmitidas a través de los tendones actuantes en cada falange, como las fuerzas de reacción transmitidas por las falanges o el metacarpo adyacentes, como también de fuerzas externas aplicadas; identificadas y situadas estas fuerzas y momentos, para cada falange podemos plantear dos ecuaciones de equilibrio de fuerzas (el modelo es bidimensional), y una 


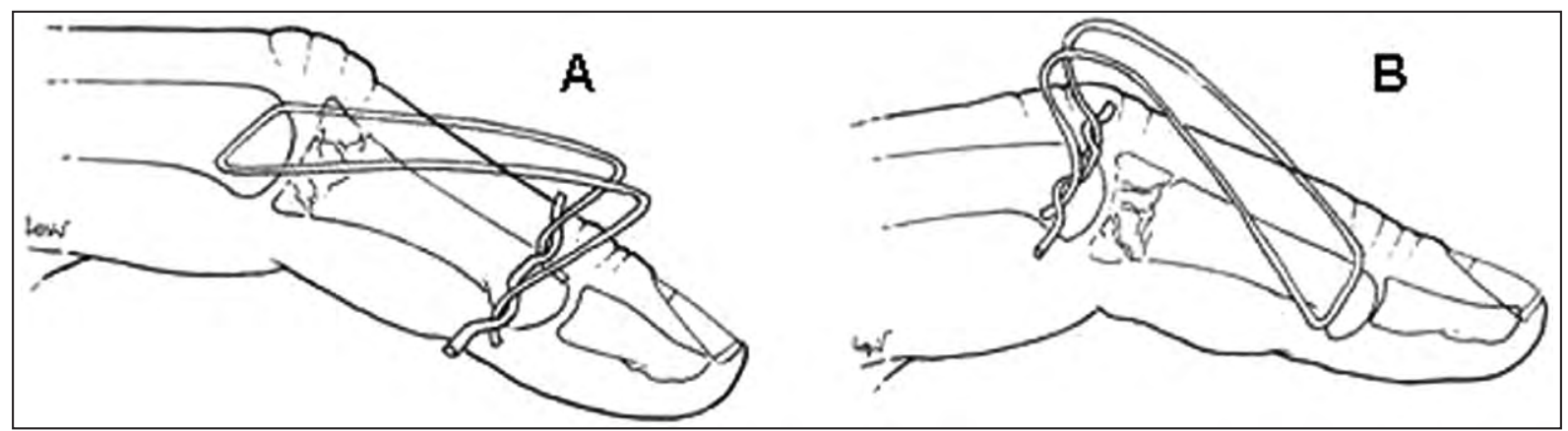

Figura 3. Distintas posibilidades de giro A. Distal, B. Proximal.

ecuación de equilibrio de momentos o pares (en total tres ecuaciones escalares). Como en conjunto disponemos de tres ecuaciones escalares por cada falange, plantearemos distintas hipótesis dando valores arbitrarios a ciertas fuerzas y momentos, de tal modo que sólo tres de ellas queden como incógnitas - las fuerzas soportadas en cada articulación siempre son incógnitas-; comenzando por la falange distal, resolveremos las ecuaciones de equilibrio de esta falange y de la falange media calculando estas incógnitas (no es necesario resolver las ecuaciones de equilibrio de la falange proximal porque las fuerzas en la articulación IFP se pueden obtener de las ecuaciones de equilibrio de la falange media). Estos cálculos se hacen para distintas hipótesis de carga, estudiando el papel jugado por cada tendón, y también variando la posición en la que es colocado el tutor. En todos los casos se comprueba que tanto la suma de fuerzas como de momentos sobre cada una de las dos falanges consideradas son nulas, con lo que se satisfacen las condiciones de equilibrio.

Esta metodología, utilizada habitualmente para resolver problemas de control en robots y brazos articulados y computacionalmente, es más eficiente que la resolución mediante métodos basados en consideraciones energéticas. Craig $^{8}$ expone la descripción completa de dicha metodología.

Todo el proceso de cálculo se ha hecho utilizando una hoja de cálculo Microsoft Excel, en la que se han tomado como datos de entrada todos los valores geométricos, incluidos los ángulos de articulación que definen la posición del dedo a estudiar, y algunas fuerzas y momentos. La hoja calcula las fuerzas y momentos que han quedado como incógnitas. Estos cálculos se han «automatizado» mediante un pequeño programa escrito en Matlab (Matworks. http:/www.mathworks.es), programa que va cambiando la posición de colocación del tutor, resuelve las ecuaciones de equilibrio de la hoja de cálculo, y representa las fuerzas calculadas en función de la posición de colocación del tutor, dando la representación gráfica correspondiente (Tablas I-III).

El cálculo se hace con tres hipótesis distintas, que permiten comparar el efecto del distractor: la primera hipótesis corresponde a la ausencia de distractor, la segunda corresponde al caso del distractor que permite el giro libremente en su inserción en la falange media (ausencia de par ó momento); y la tercera hipótesis corresponde al caso del distractor que impide el giro en su punto de inserción en la falange media (aparición de par ó momento); esta tercera hipótesis es equivalente a permitir el giro del distractor libremente en la inserción de la falange proximal. (Figura 3)

El resultado fundamental de los cálculos es la componente Y de la fuerza de reacción en la articulación IFP, que nos dice cual será la tendencia a la luxación de la falange media en cada caso. Si este valor es positivo, indica que la articulación debe hacer una fuerza positiva (Y) para evitar la luxación de dicha articulación (la falange tendería a luxarse hacia el lado palmar). En cambio, si dicho valor es negativo, indica que la luxación tendería a producirse en sentido dorsal de la mano. Además, el valor absoluto de dicha fuerza indica si la tendencia a la luxación es mayor o menor. 
En este trabajo hemos estudiado tres posiciones del dedo diferentes:

a) Extensión completa (ángulos de articulación todos nulos)

b) Posición intermedia (ángulos de articulación de $10^{\circ}, 45^{\circ}$ y $10^{\circ}$ )

c) Flexión completa (ángulos de articulación de $90^{\circ}, 90^{\circ}$ y $80^{\circ}$ )

Para cada una de estas posiciones, se ha estudiado la fuerza tendente a luxar la articulación con las distintas hipótesis de colocación y posición del tutor.

\section{RESULTADOS}

Los resultados de cada una de estas tres posiciones, analizando la componente $\mathrm{Y}$ de la reacción en la articulación IFP, se muestran a continuación:

1. Extensión completa (Tabla I).

Sin distractor hay una pequeña fuerza que tiende a luxar el dedo en sentido palmar.

Con distractor:

a) permitiendo el giro distal, colocado en la parte media de la falange no hay tendencia luxante, por el contrario colocado más distalmente a este punto hay una tendencia muy pequeña a luxar palmarmente, tendencia que se invierte al colocar el punto de giro en la primera mitad de la falange, es decir más proximalmente.

TABLA I - FUERZA DE DISLOCACIÓN FRENTE A POSICIÓN DE COLOCACIÓN DEL DISTRACTOR EN ARTICULACIÓN IFP. POSICIÓN DE EXTENSIÓN COMPLETA

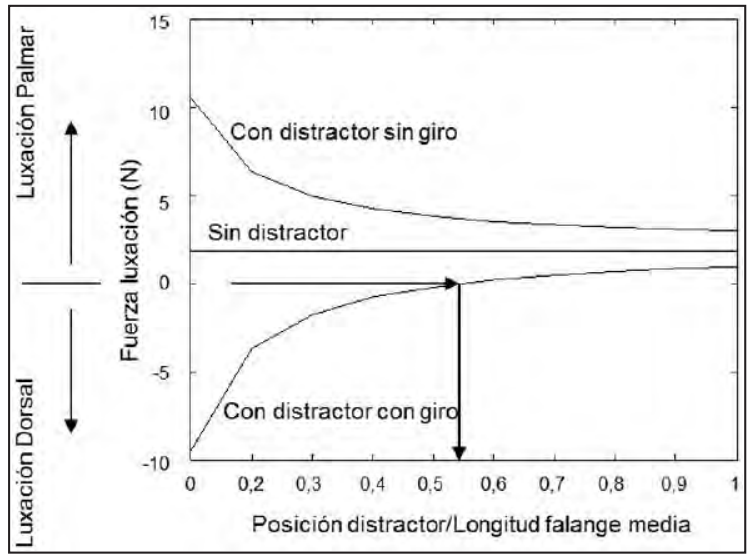

TABLA II - FUERZA DE DISLOCACIÓN FRENTE A POSICIÓN DE COLOCACIÓN DEL DISTRACTOR EN ARTICULACIÓN IFP. POSICIÓN INTERMEDIA ENTRE EXTENSIÓN COMPLETA Y FLEXIÓN COMPLETA

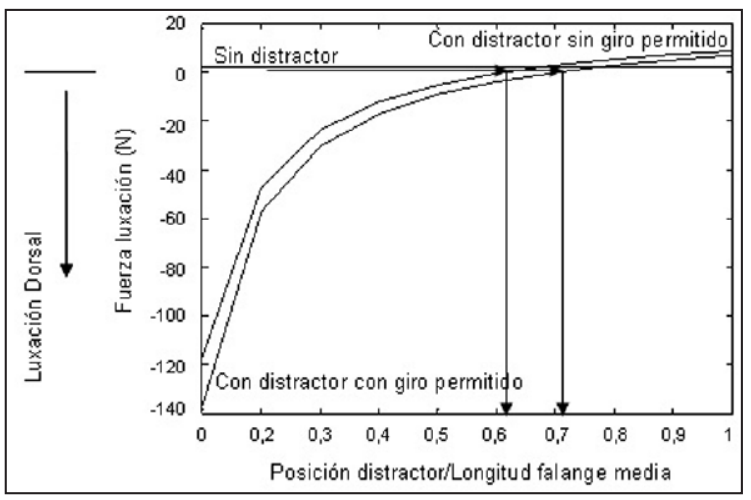

TABLA III - Fuerza dE dIsLocación FRENTE A POSICIÓN DE COLOCACIÓN DEL DISTRACTOR EN ARTICULACIÓN IFP. POSICIÓN DE FLEXIÓN COMPLETA

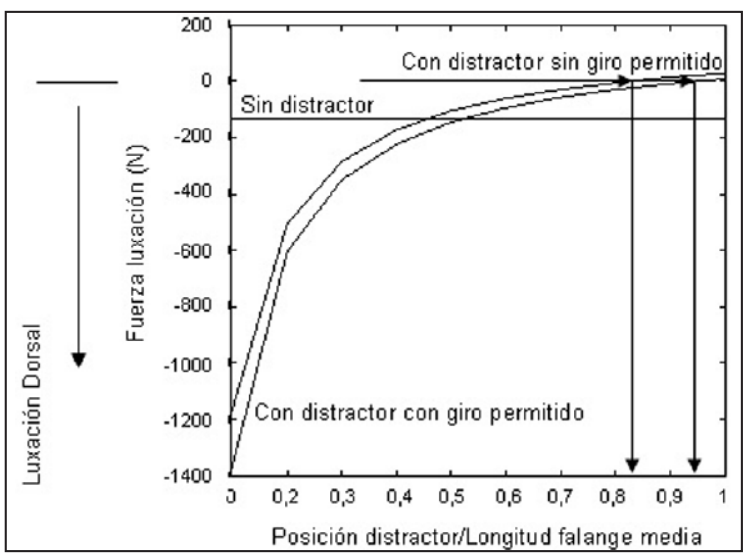

b) sin permitir el giro en la inserción distal, siempre hay tendencia a la luxación palmar, más pequeña cuanto más distalmente se coloque.

2. Posición intermedia (Tabla II).

Sin distractor se mantiene una pequeña tendencia luxante palmar.

Con distractor no hay tendencia a luxarse, emplazando el punto de giro aproximadamente al $72 \%$ de la distancia (permitiendo giro distal), y al $62 \%$ de la distancia (no permitiendo el giro distal).

Si se emplaza más proximalmente, la tendencia es hacia luxación dorsal, mayor cuanto más proximalmente se coloque. 


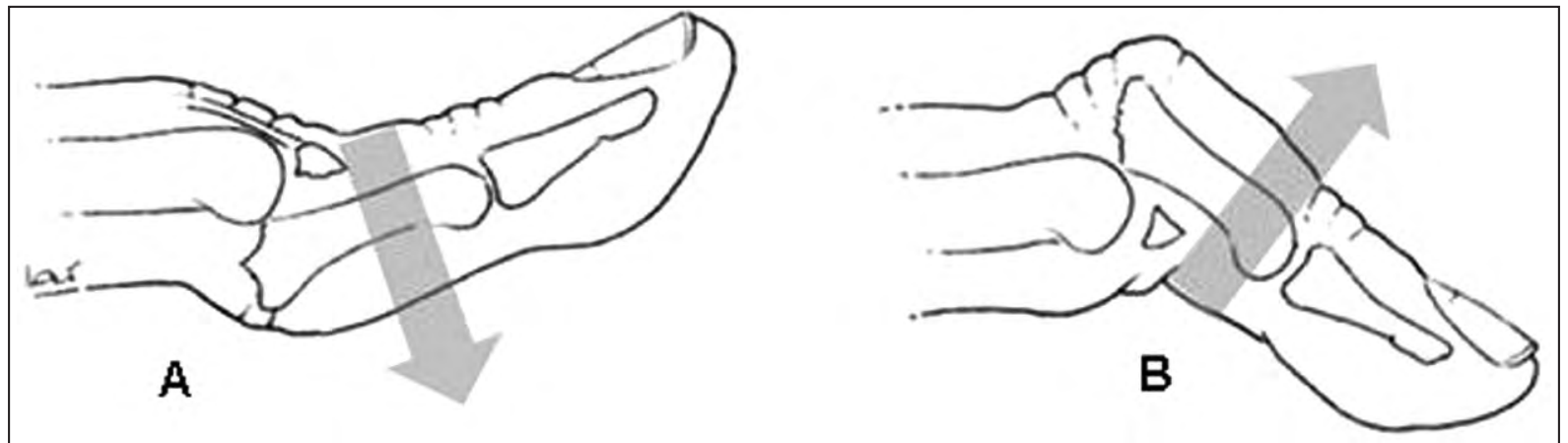

Figura 4. A. Fractura luxación volar, tiene una tendencia a la luxación volar pero presentan generalmente una estabilidad dorsal procurada por las partes blandas del dorso, lo que ocurre si no se acompaña de un fragmento dorsal por arrancamiento de la bandeleta central extensora. B. Al contrario la luxación dorsal, suele presentar una tendencia estabilizadora volar, que no suele suceder si además presenta un fragmento por arrancamiento de la placa volar.

\section{Flexión completa (Tabla III).}

Sin distractor hay tendencia a luxar dorsalmente.

Con distractor la tendencia a luxar dorsalmente es tanto mayor cuanto más proximal es la inserción, como demuestran las curvas de la tabla. No hay tendencia a luxar emplazando el punto de giro aproximadamente al 95\% de la distancia (permitiendo giro distal), y al $82 \%$ de la distancia (no permitiendo el giro distal).
Si se emplaza más proximalmente, la tendencia es hacia luxación dorsal, mayor cuanto más proximalmente se coloque.

\section{DISCUSIÓN}

Este modelo matemático aclara el comportamiento biomecánico de las fracturas conminutas de la base de la F2, y conlleva importantes impli-

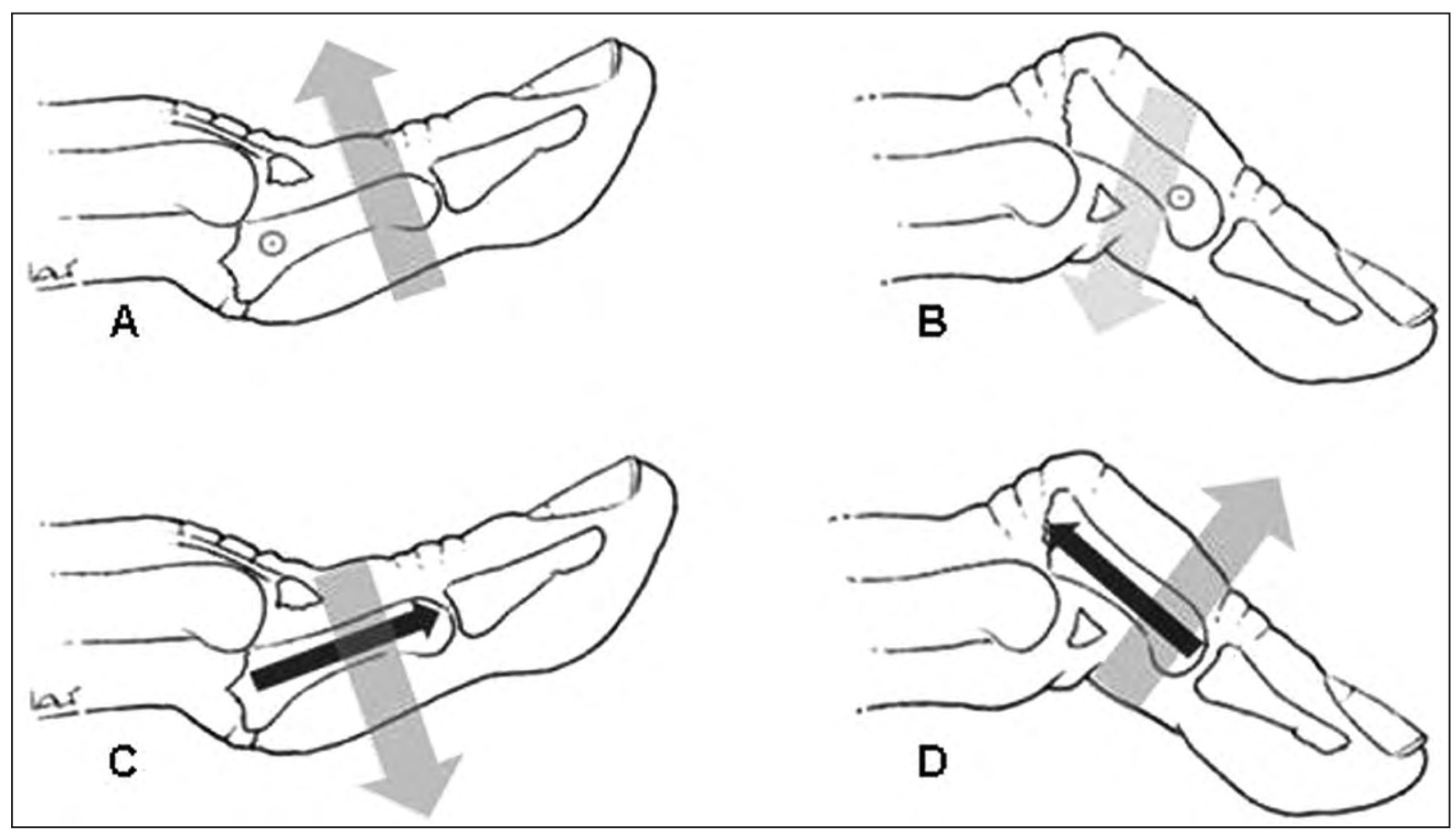

Figura 5. A. Tendencia estabilizadora con la ubicación de la aguja resistente próxima a la articulación en fracturas inestables volarmente. $B$. Tendencia estabilizadora con la ubicación de la aguja resistente lejos de la articulación. C. Tendencia luxante mayor alejándose de la articulación en inestabilidades volares. D. Tendencia luxante mayor aproximándose a la articulación en inestabilidades dorsales. 


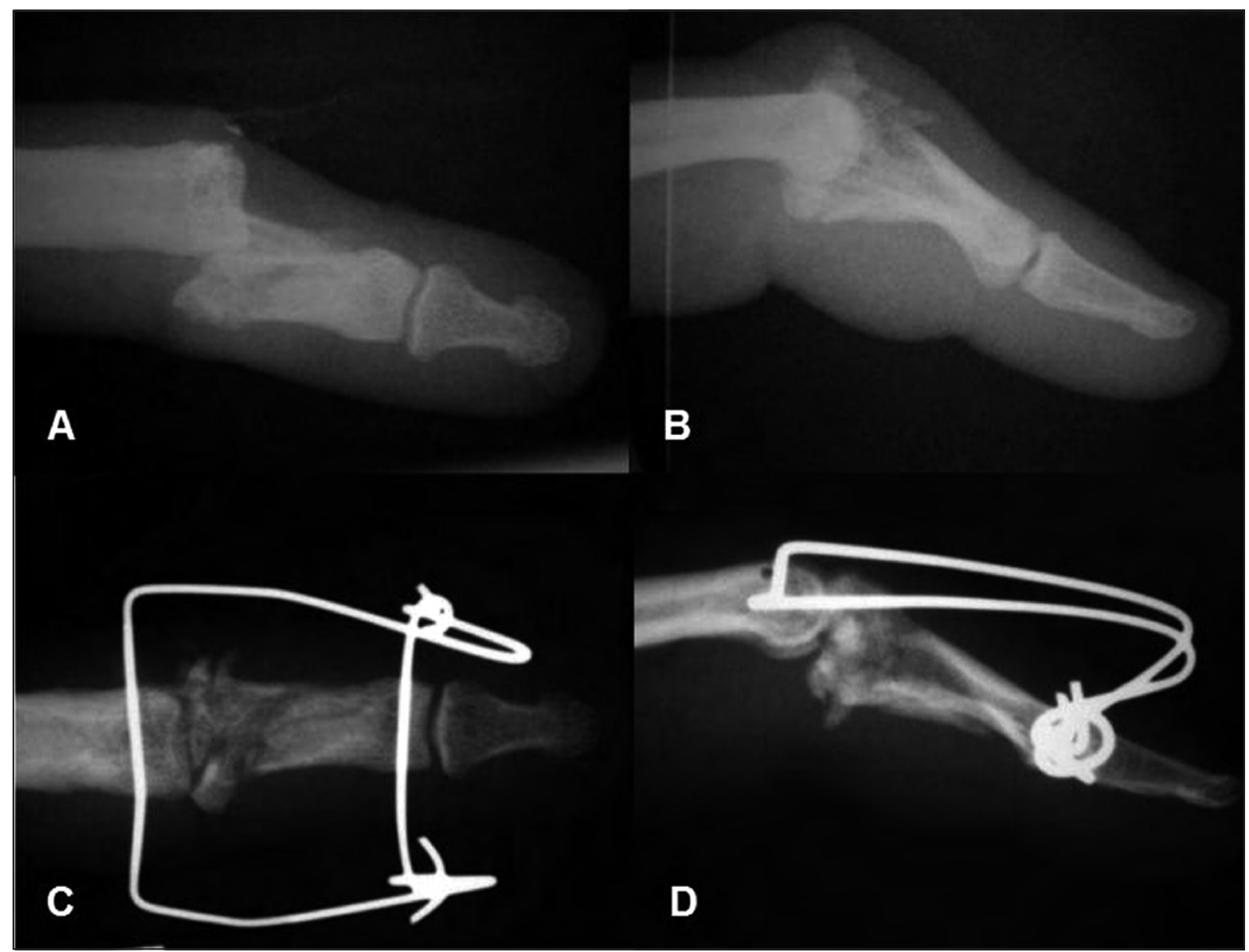

Figura 6. A y B. Imagen radiográfica de una fractura conminuta de IFP. C y D. Una vez colocado el distractor dinámico.

caciones clínicas. En lesiones con arrancamientos de la placa volar, se verifica la tendencia a la luxación dorsal de la falange. Cuanto más distal a la articulación se coloque la aguja, mayor será la tendencia a la inestabilidad. Por esta razón, recomendamos la inserción cerca de la articulación IFP. En las lesiones con arrancamiento dorsal, la tendencia es contraria con luxación hacia palmar. La inestabilidad será mayor cuanto más próxima de la articulación este colocada la aguja. La recomendación en este caso para obtener mayor estabilización, será la contraria; es decir colocar la aguja lo más distalmente posible.

Hasta el momento la literatura sobre el sistema de artrodiástasis funcional aquí analizado no recoge indicaciones sobre la posición óptima de las agujas ${ }^{4,5}$. A este detalle técnico hasta ahora no se le había prestado atención. Lo importante hasta ahora era la funcionalidad mantener una movilización precoz y no se había reparado en la tendencia estabilizadora relacionada con la ubicación de las agujas. En este sentido, pensamos que nuestro trabajo aporta una constatación matemática de los factores de contención de la articulación con importante aplicación clínica.

Una limitación de este estudio es que se trata de una modelización matemática que no tiene en cuenta algún otro factor relacionado con la clínica. No obstante, en nuestra experiencia el comportamiento clínico se ajusta bien a los datos matemáticos obtenidos sirviéndonos como referencia a la hora de colocar las agujas.

La observación en clínica con fracturas conminutas de la IFP a las que hemos aplicado este tratamiento, nos reafirma en que la comprensión de este modelo nos capacita para entender y cambiar la evolución de estas graves fracturas articulares (Figura 4).

La ubicación de la aguja distal dependiendo del tipo de inestabilidad que tengamos puede 


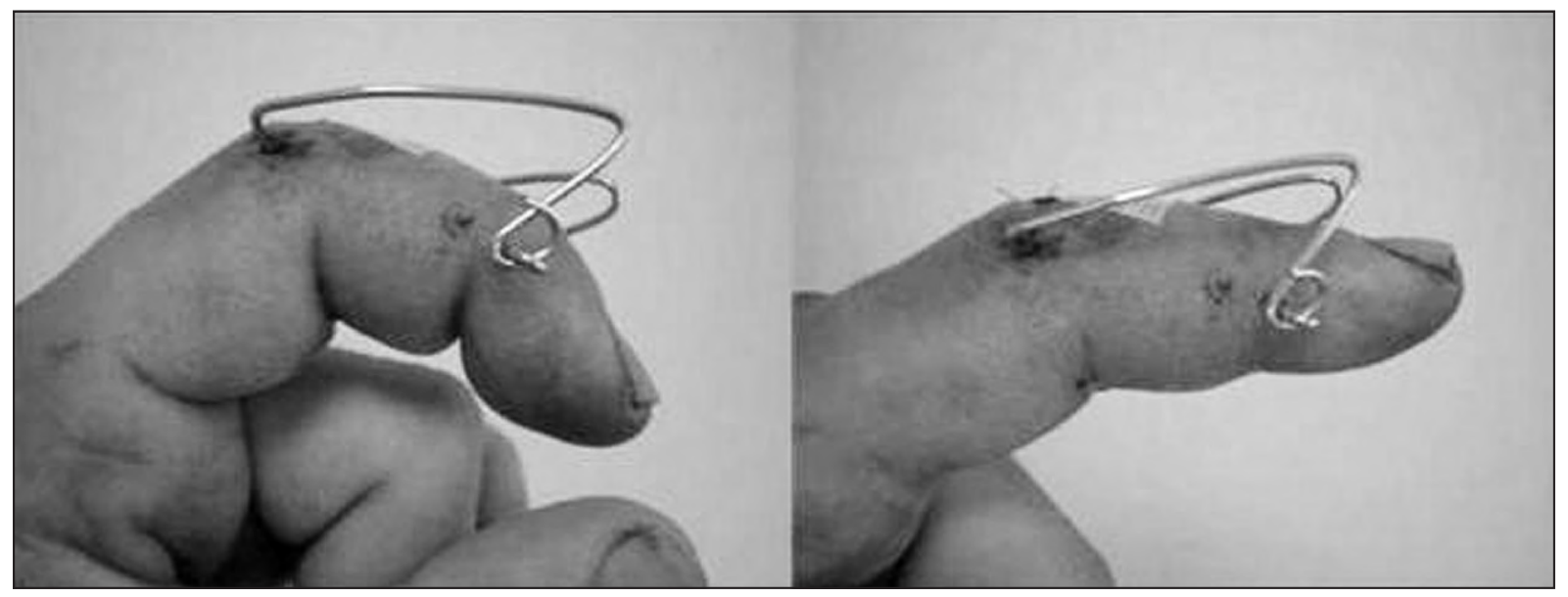

Figura 7. Imagen clínica del caso anterior.

contrarrestar la tendencia luxante. Así, aproximarse a la articulación estabiliza hacia dorsal, alejarse de la articulación estabiliza hacia volar.

\section{(Figura 5)}

En las Figuras 6 y 7 se presenta un caso de fractura conminuta de la articulación IFP; obsérvese como con el movimiento poco a poco va llevando los fragmentos por ligamentotaxis a su sitio y se recompone la articulación.

\section{CONCLUSIÓN}

Para la aplicación clínica de los resultados aquí obtenidos, es primordial la exploración previa de la estabilidad articular. Una vez determinada ésta, se procederá a la colocación de la aguja resistente alejándose de la articulación IFP en la inestabilidad dorsal, aproximándose de la articulación IFP en la inestabilidad volar.
1. Mir Bullo X, Lluch Bergadá A. Luxaciones y traumatismos articulares de la mano. En: Manual SECOT de Cirugía Ortopédica y Traumatología. $2^{\mathrm{a}}$ ed. Madrid: Ed. Panamericana 2010: 913-20.

2. Agee JM. Unstable fracture dislocations of the proximal interphalangeal joint. Treatment with the force couple splint. Clin Orthop, 1987; 214: 101-12.

3. Allison DM. Fractures of the base of the middle phalanx treated by a dynamic external

\section{BIBLIOGRAFÍA}

fixation device. J Hand Surg B, 1996; 21: 305-10.

4. Bindra RR. Dislocation and fractures dilocations of the metacarpophalangeal and proximal interphalangeal joint. En: Ring DC, Cohen MS (Eds). Fractures of the Hand and Wrist, $1^{\mathrm{a}}$ ed, New York: Ed Informa Healthcare USA, Inc, 2007: 41-73.

5. Giddins GEB, Hynes MC. Dynamic external fixation for pilon fractures of the interphalangeal joint. J Hand Surg Br, 2001; 26:122-4.
6. Suzuki Y, Matsunaga T, Sato S, Yokoi T. The pins and rubbers traction system for treatment of comminuted intraarticular fractures and fracture-dislocations in the hand. J Hand Surg $\mathrm{Br}$, 1994; 19: 98-107.

7. Chao E, An KN, Cooney W, Linscheid RL. Biomechanics of the Hand. A Basic Research Study. Singapore: World Scientific Publishing Co., 1989.

8. Craig JJ. Introduction to Robotics. Mechanics and control, 2nd edition, New York: Ed. Addison-Wesley Inc., 1989. 